\title{
Acute glaucoma presenting with abdominal symptoms
}

\author{
N J Watson, G R Kirkby
}

The patient with abdominal pain and a red eye may have glaucoma
Birmingham and Midland Eye Hospital, Birmingham B3 2NS

N J Watson, FRCs, senior house officer

G R Kirkby, FRCS, consultant ophthalmic surgeon

\section{Correspondence to:}

Mr Kirkby.

BrMed f 1989;299:254
Acute angle closure glaucoma is a rare condition that affects $0 \cdot 1 \%$ of the population aged over 50 years. ${ }^{12}$ Patients usually present with sudden onset of severe pain in or over the affected eye and reduced vision. They may have had prodromal attacks of aching, blurred vision, and haloes round lights, particularly at night. The eye is red, with oedema of the cornea and a fixed semidilated pupil, and is hard when palpated.

Rarely patients present not with severe eye pain but with abdominal symptoms predominating that result from effects of an appreciable rise in intraocular pressure. The symptoms are prostration, nausea, vomiting, and pain. ${ }^{3}$ Patients and their doctors may therefore ignore serious eye disease.

We describe two patients who illustrate this unusual presentation of the disease.

\section{Case 1}

An 86 year old woman with senile dementia was admitted to hospital with increasing confusion, nausea, vomiting, and colicky lower abdominal pain. Urinary tract infection was diagnosed. She was prescribed systemic antibiotics and chloramphenicol eye drops for her left eye, which was red.

Four days later the abdominal pain had not settled, no abdominal abnormality was found, and a midstream urine sample (taken before she started taking antibiotics) showed no evidence of infection. The red eye had not resolved, so an opinion from an ophthalmologist was requested. Her visual acuities were $6 / 18$ right and count fingers left. The left eye had a fixed semidilated pupil. The intraocular pressure was $6 \cdot 1 \mathrm{kPa}$ (normal range up to $2 \cdot 8 \mathrm{kPa}$ ). The fundus could not be seen. Acute angle closure glaucoma was diagnosed.

The intraocular pressure returned to normal with medical treatment, and concurrently the abdominal pain resolved. Medical treatment was followed by prophylactic peripheral iridotomy on the right eye with a neodymium yttrium aluminium garnet laser and drainage surgery to the left eye. Her vision, however, did not improve.

\section{Case 2}

A 74 year old woman with senile dementia developed increasing confusion, nausea, abdominal pain, and frequency of micturition. Urinary tract infection was diagnosed at her day hospital and by her general practitioner. Her right eye was uncomfortable and conjunctivitis suspected. She was prescribed systemic antibiotics and chloramphenicol eye drops. Two days later the left eye became red, and it was assumed that the conjunctivitis had spread. Chloramphenicol was then prescribed for both eyes. A midstream urine specimen contained no cells and grew no organisms.

Two days later she fell and broke her arm, and it was established that this was the result of poor eyesight. An opinion was sought from an ophthalmologist. Her visual acuities were hand movements right and count fingers left. She had oedema of the cornea with fixed semidilated pupils in both eyes. The intraocular pressures were $9.6 \mathrm{kPa}$ right and $7.9 \mathrm{kPa}$ left. The fundus was not visible. Bilateral acute angle closure glaucoma was diagnosed.

The intraocular pressures returned to normal with medical treatment, and the abdominal symptoms resolved. Later she had drainage surgery to both eyes. Her vision did not improve, and she remained blind.

\section{Discussion}

An early diagnosis of acute glaucoma is essential because a prolonged rise in intraocular pressure can result in permanent loss of vision due to ischaemia of the optic nerve. In addition, the condition affects both eyes in nearly two thirds of patients, ${ }^{1}$ and this can be prevented by early treatment by peripheral iridectomy or iridotomy.

The cases of the two patients reported here show that it is important to consider acute glaucoma when a patient presents with nausea, abdominal pain, and vomiting and a painless red eye. This is especially true in elderly patients who may be too confused or debilitated to give a good clinical history and in whom the abdominal symptoms may predominate.

1 Kilker A, Hetherington J Jr. Becker-Shaffer's diagnosis and management of the laucomas. 4th ed St Louis: Mosby, 1976:183.

2 Spaeth GL. The normal development of the human anterior chamber angle: a new system of descriptive grading. Trans Ophthalmol Soc UK 1971;91:709. 3 Rains AJH, Richie HD, eds. Bailey and Love's short practice of surgery. 17th ed. London: Lewis, 1973

(Accepted 17 April 1989)

\section{ANY QUESTIONS}

Are there any long term side effects from antacids containing aluminium?

It is now accepted that aluminium may be absorbed into the body via the gut. In patients with impaired kidney function aluminium accumulation results in three syndromes-namely, dialysis encephalopathy, osteomalacic renal osteodystrophy, and microcytic anaemia. Whether aluminium accumulation occurs in the presence of normal kidney function is controversial. With a normal diet $0 \cdot 1-0 \cdot 3 \%$ of dietary aluminium is absorbed, such low absorption being almost at the limits of sensitivity of methods of measuring aluminium. Aluminium absorption is, however, detectable after modest doses of compounds containing 125-464 mg aluminium daily. Antacids containing aluminium may increase the daily intake of aluminium by $840-5000 \mathrm{mg}$ a day depending on the choice of antacid. There are no long term studies of aluminium accumulation in patients other than those with impaired renal function taking antacids containing aluminium. In 1985 the United States Food and Drug Administration decided to make no change to the labelling on over the counter antacids containing aluminium because the available data showed that aluminium toxicity was not a problem either in normal people or in patients with mild or moderate renal impairment. The labelling now warns against continued use for more than two weeks. - T K DANESHMEND, clinical lecturer in therapeutics, Nottingham

Lione A Aluminium toxicity and aluminium containing medications. Pharmacol Ther 1985;29. 255-85.

Parkinson IS, Ward MK. Aluminium toxicity. In: Turner P, Volans GN, eds. Recent advances in clinical pharmacology and toxicology. No 4. Edinburgh: Churchill Livingstone, 1989:219-32. 